\title{
SKEW SYMMETRIC NORMAL OPERATORS
}

\author{
CHUN GUANG LI AND SEN ZHU
}

(Communicated by Marius Junge)

\begin{abstract}
An operator $T$ on a complex Hilbert space $\mathcal{H}$ is said to be skew symmetric if there exists a conjugate-linear, isometric involution $C: \mathcal{H} \longrightarrow \mathcal{H}$ so that $C T C=-T^{*}$. In this paper, we shall give two structure theorems for skew symmetric normal operators.
\end{abstract}

\section{INTRODUCTION}

In linear algebra, there is a lot of work on the theory of symmetric matrices (that is, $T=T^{t}$ ) and skew symmetric matrices (that is, $T+T^{t}=0$ ), which have many motivations in function theory, matrix analysis and other mathematical disciplines. They have many applications even in engineering disciplines. Many important results related to canonical forms for symmetric matrices or skew symmetric matrices are obtained in 4, 11, 15. In particular, L.-K. Hua 12 proved that each skew symmetric matrix can be written as $A=U B U^{t}$, where $U$ is a unitary matrix and

$$
B=\left[\begin{array}{cc}
0 & \lambda_{1} \\
-\lambda_{1} & 0
\end{array}\right] \oplus\left[\begin{array}{cc}
0 & \lambda_{2} \\
-\lambda_{2} & 0
\end{array}\right] \oplus \cdots \oplus\left[\begin{array}{cc}
0 & \lambda_{r} \\
-\lambda_{r} & 0
\end{array}\right] \oplus 0 \oplus \cdots \oplus 0 .
$$

Generalizing the notion of complex symmetric matrices, Garcia and Putinar 6 initiated the study of complex symmetric operators on Hilbert spaces. To proceed, we first introduce some notation and terminologies. In this paper, $\mathcal{H}, \mathcal{K}, \mathcal{K}_{1}, \mathcal{K}_{2}, \cdots$ will always denote complex separable Hilbert spaces. We let $\mathcal{B}(\mathcal{H})$ denote the algebra of all bounded linear operators on $\mathcal{H}$.

Definition 1.1. A map $C$ on $\mathcal{H}$ is called an antiunitary operator if $C$ is conjugatelinear, invertible and $\langle C x, C y\rangle=\langle y, x\rangle$ for all $x, y \in \mathcal{H}$. If, in addition, $C^{-1}=C$, then $C$ is called a conjugation.

Definition 1.2. An operator $T \in \mathcal{B}(\mathcal{H})$ is said to be complex symmetric if there exists a conjugation $C$ on $\mathcal{H}$ such that $C T C=T^{*}$.

Many important operators, such as Hankel operators, the Volterra integration operator, truncated Toeplitz operators, normal operators and binormal operators,

Received by the editors September 17, 2011 and, in revised form, October 30, 2011.

2010 Mathematics Subject Classification. Primary 47B25, 47B15; Secondary 47A65.

Key words and phrases. Skew symmetric operators, complex symmetric operators, normal operators.

This work was supported by NNSF of China (11101177, 10971079, 11271150), China Postdoctoral Science Foundation (2011M500064, 2012T50392), Shanghai Postdoctoral Scientific Program (12R21410500), and Science Foundation for Young Teachers of Northeast Normal University (12QNJJ001).

The authors wish to thank the editor and the referee for many helpful comments and suggestions which greatly improved the manuscript. 
have proved to be complex symmetric $([6,7,10])$. A lot of important work has been done concerning the structure of complex symmetric operators (see [6, 7, 8, 5, , 9, 2 , 10, 19, 20]). However, less attention has been paid to skew symmetric operators.

Definition $1.3([17)$. An operator $T \in \mathcal{B}(\mathcal{H})$ is said to be skew symmetric if there exists a conjugation $C$ on $\mathcal{H}$ such that $C T C=-T^{*}$.

Using [6, Lemma 1], one can easily deduce that $T \in \mathcal{B}(\mathcal{H})$ is skew symmetric if and only if there exists an orthonormal basis (ONB for short) $\left\{e_{n}\right\}$ of $\mathcal{H}$ such that $\left\langle T e_{n}, e_{m}\right\rangle=-\left\langle T e_{m}, e_{n}\right\rangle$ for all $m, n$; that is, $T$ admits a skew symmetric matrix representation with respect to $\left\{e_{n}\right\}$. In [17, Zagorodnyuk studied the polar decomposition of skew symmetric operators and obtained some basic properties of skew symmetric operators. In [18, Zagorodnyuk studied the skew symmetry of cyclic operators.

Now we list some elementary facts about skew symmetric operators. The proofs are simple and left to the reader.

Lemma 1.4. Let $C$ be a conjugation on $\mathcal{H}$. Denote $S_{C}(\mathcal{H})=\{X \in \mathcal{B}(\mathcal{H}): C X C=$ $\left.-X^{*}\right\}$. Then:

(i) if $A, B \in \mathcal{B}(\mathcal{H}), C A C=A^{*}$ and $C B C=B^{*}$, then $[A, B]:=A B-B A \in$ $S_{C}(\mathcal{H})$

(ii) if $T \in S_{C}(\mathcal{H})$, then $C T^{2 n} C=\left(T^{2 n}\right)^{*}$ for all $n \in \mathbb{N}$;

(iii) the class $S_{C}(\mathcal{H})$ is norm-closed and forms a Lie algebra under the commutator bracket $[\cdot, \cdot]$;

(iv) if $T \in S_{C}(\mathcal{H})$, then $\sigma(T)=-\sigma(T)$.

Let us first see some concrete examples.

Example 1.5. Assume that $T \in \mathcal{B}\left(\mathbb{C}^{3}\right)$ admits the following representation:

$$
T=\left[\begin{array}{ccc}
1 & 0 & 0 \\
0 & -1 & 0 \\
0 & 0 & -1
\end{array}\right] \begin{aligned}
& e_{1} \\
& e_{2}, \\
& e_{3}
\end{aligned}
$$

where $\left\{e_{1}, e_{2}, e_{3}\right\}$ is an ONB of $\mathbb{C}^{3}$. Then $T$ is not a skew symmetric operator.

Since the trace of a skew symmetric matrix is zero and the trace is invariant under the unitary transform, taking the trace shows that $T$ is not a skew symmetric operator.

Example 1.6. Assume that $T \in \mathcal{B}\left(\mathbb{C}^{2}\right)$ admits the following representation:

$$
T=\left[\begin{array}{ll}
0 & 1 \\
0 & 0
\end{array}\right] \quad \begin{aligned}
& e_{1} \\
& e_{2}
\end{aligned}
$$

where $\left\{e_{1}, e_{2}\right\}$ is an ONB of $\mathbb{C}^{2}$. Then $T$ is not a skew symmetric operator.

In fact, if not, then there exists a conjugation $C$ on $\mathbb{C}^{2}$ such that $C T C=-T^{*}$. Hence $C e_{1}=\alpha e_{2}$ for some $\alpha \in \mathbb{C}$ with $|\alpha|=1$. It follows that $C T C e_{1}=\bar{\alpha} C T e_{2}=$ $\bar{\alpha} C e_{1}=e_{2}$ and $-T^{*} e_{1}=-e_{2}$, a contradiction.

The two examples above illustrate that the spectral condition " $\sigma(T)=-\sigma(T)$ " is in general not sufficient for a (normal) operator $T$ to be skew symmetric. If $T \in \mathcal{B}(\mathcal{H})$ and $C$ is a conjugation on $\mathcal{H}$ satisfying $C T C=-T^{*}$, then $C(T-$ $\lambda) C=-(T+\lambda)^{*}$ and $C(T+\lambda)^{*} C=-(T-\lambda)$ for $\lambda \in \mathbb{C}$. Thus we obtain $\operatorname{dim} \operatorname{ker}(T-\lambda)=\operatorname{dim} \operatorname{ker}(T-\lambda)^{*}$. This shows that multiplicity is an important invariant in the structure of skew symmetric operators. 
Example 1.7. Assume that $T \in \mathcal{B}\left(\mathbb{C}^{3}\right)$ admits the following representation:

$$
T=\left[\begin{array}{lll}
0 & 1 & 0 \\
0 & 0 & 1 \\
0 & 0 & 0
\end{array}\right] \begin{aligned}
& e_{1} \\
& e_{2}, \\
& e_{3}
\end{aligned}
$$

where $\left\{e_{1}, e_{2}, e_{3}\right\}$ is an ONB of $\mathbb{C}^{3}$. Then $T$ is a skew symmetric operator.

For $x=\alpha_{1} e_{1}+\alpha_{2} e_{2}+\alpha_{3} e_{3}$, define $C x=-\overline{\alpha_{3}} e_{1}+\overline{\alpha_{2}} e_{2}-\overline{\alpha_{1}} e_{3}$. Then it can be verified that $C$ is a conjugation on $\mathbb{C}^{3}$. Also, a direct calculation shows that $C T C=-T^{*}$. Hence $T$ is skew symmetric. Set

$$
U=\left[\begin{array}{ccc}
\frac{1}{\sqrt{2}} & 0 & \frac{i}{\sqrt{2}} \\
0 & 1 & 0 \\
-\frac{1}{\sqrt{2}} & 0 & \frac{i}{\sqrt{2}}
\end{array}\right] e_{1} e_{2} .
$$

It is easy to check that $U$ is a unitary operator and

$$
U^{*} T U=\left[\begin{array}{ccc}
0 & \frac{1}{\sqrt{2}} & 0 \\
-\frac{1}{\sqrt{2}} & 0 & \frac{i}{\sqrt{2}} \\
0 & -\frac{i}{\sqrt{2}} & 0
\end{array}\right] e_{2} e_{2} .
$$

Likewise, one can prove that each operator induced by a Jordan block with odd order is skew symmetric.

It is straightforward to see that each complex symmetric operator is biquasitriangular (see [6, Proposition 1]). This is not the case for skew symmetric operators. Here is an example.

Example 1.8. Let $S$ be the unilateral shift on $\mathcal{H}$ defined by $S e_{i}=e_{i+1}$ for $i \in \mathbb{N}$, where $\left\{e_{i}\right\}_{i \in \mathbb{N}}$ is an ONB of $\mathcal{H}$. Set

$$
T=\left[\begin{array}{cc}
S+I & 0 \\
0 & -S^{*}-I
\end{array}\right] \begin{aligned}
& \mathcal{H} \\
& \mathcal{H}
\end{aligned} .
$$

Then $T$ is skew symmetric.

In fact, we can define a conjugation $C$ on $\mathcal{H}$ by $C\left(\sum_{i} \alpha_{i} e_{i}\right)=\sum_{i} \overline{\alpha_{i}} e_{i}$ for $\sum_{i} \alpha_{i} e_{i} \in \mathcal{H}$. Set

$$
D=\left[\begin{array}{ll}
0 & C \\
C & 0
\end{array}\right] \mathcal{H}
$$

Then $D$ is a conjugation on $\mathcal{H} \oplus \mathcal{H}$ and one can verify that $D T D=-T^{*}$. It is easy to see that $T-I$ is a Fredholm operator and ind $(T-I)=$ ind $S+$ ind $\left(-S^{*}-2 I\right)=-1$. Hence $T$ is skew symmetric but not a biquasitriangular operator.

This paper was inspired by [9] and [19], where the complex symmetry of partial isometry and weighted shifts is studied. In view of various results on complex symmetric operators, it is natural to study analogously the structure of skew symmetric operators. There are several motivations for our investigation.

For one thing, skew symmetric matrices have many applications in pure mathematics, applied mathematics and even in engineering disciplines. Real skew symmetric matrices are very important in applications, including function theory [11, 12, the solution of linear quadratic optimal control problems, robust control problems, model reduction, crack following in anisotropic materials and others (see [1, 13, 14, 16]). In view of these applications, it is natural to investigate skew symmetric operators in the setting of a Hilbert space. 
Another reason for studying skew symmetric operators is their close relation to complex symmetric operators. Through Lemma1.4(i)/(ii), one may see this point. It is often difficult to determine whether a given operator is complex symmetric. Lemma 1.4 (ii) provides an approach to construct new complex symmetric operators. On the other hand, each operator $T$ on $\mathcal{H}$ can be written as the sum of a complex symmetric operator and a skew symmetric operator. In fact, arbitrarily choose a conjugation $C$ on $\mathcal{H}$ and set $A=\frac{1}{2}\left(T+C T^{*} C\right), B=\frac{1}{2}\left(T-C T^{*} C\right)$. Then $A$ is complex symmetric, $B$ is skew symmetric and $T=A+B$. In a certain sense, this reflects some universality of complex symmetric and skew symmetric operators. By the following result, one can use complex symmetric operators to construct skew symmetric operators.

Proposition 1.9. If $A \in \mathcal{B}(\mathcal{H})$ is complex symmetric, then $T=A \oplus(-A)$ is skew symmetric.

Proof. Since $A$ is complex symmetric, there exists a conjugation $C$ on $\mathcal{H}$ such that $C T C=T^{*}$. Set

$$
D=\left[\begin{array}{ll}
0 & C \\
C & 0
\end{array}\right] \mathcal{H}
$$

It is easy to see that $D$ is a conjugation on $\mathcal{H} \oplus \mathcal{H}$ and

$$
D T D=(-C A C) \oplus(C A C)=\left(-A^{*}\right) \oplus A^{*}=-T^{*} .
$$

Hence $T$ is skew symmetric.

Also, it can be seen from Lemma 1.4 (iii) that skew symmetric operators may have some internal connections to the Lie algebra of operators. All of the abovementioned connections constitute partial sources of skew symmetric operators in the Hilbert space setting.

The main aim of this paper is to give a characterization of normal operators which are skew symmetric. In fact, we shall give two structure theorems for skew symmetric normal operators. In what follows, we denote $\Sigma=\{\alpha \in \mathbb{C}: \operatorname{Im} \alpha>$ $0\} \cup\{\alpha \in \mathbb{C}: \operatorname{Im} \alpha=0, \operatorname{Re} \alpha>0\}$. Given a normal operator $N$, we denote by $E_{N}(\cdot)$ the projection-valued spectral measure corresponding to $N$.

Now we can list our main results.

Theorem 1.10 (Main Theorem 1). Let $T \in \mathcal{B}(\mathcal{H})$ be normal. Then the following are equivalent:

(i) $T$ is skew symmetric;

(ii) $\left.T\right|_{(\operatorname{ker} T)^{\perp}} \simeq N \oplus(-N)$, where $N$ is a normal operator on some Hilbert space $\mathcal{K}$ with $E_{N}(\mathbb{C} \backslash \Sigma)=0$;

(iii) $\left.T\right|_{(\operatorname{ker} T)^{\perp}} \simeq N \oplus(-N)$, where $N$ is a normal operator on some Hilbert space $\mathcal{K}$.

Here and in what follows, we let $\simeq$ denote the unitary equivalence relation of operators.

Note that each normal operator acting on finite dimensional Hilbert space has a diagonal matrix representation. Then, using Proposition 1.9 and the remark right after Example 1.6, one can see that the result of Theorem 1.10 in the finite dimensional case is obvious. However, the infinite dimensional case is not so easy, since a general normal operator $\left(M_{z}\right.$ on $L^{2}[0,1]$ for instance) does not have a diagonal matrix representation. 
Theorem 1.11 (Main Theorem 2). A normal operator $T \in \mathcal{B}(\mathcal{H})$ is skew symmetric if and only if there are mutually singular measures $\mu_{\infty}, \mu_{1}, \mu_{2}, \cdots$ (some of which may be zero) such that (1) $\mu_{j}(\sigma)=\mu_{j}(-\sigma)$ for any $1 \leq j \leq \infty$ and any Borel subset $\sigma$ of $\mathbb{C}$, and (2) $T$ is unitarily equivalent to the operator

$$
N=\bigoplus_{1 \leq j \leq \infty} N_{j}^{(j)}
$$

where $N_{j}$ is the "multiplication by $z$ " operator on $L^{2}\left(\mu_{j}\right)(1 \leq j \leq \infty)$.

\section{Proofs OF MAIN THEOREMS}

We first give some useful lemmas.

Lemma 2.1. A normal operator $T \in \mathcal{B}(\mathcal{H})$ is skew symmetric if and only if $\left.T\right|_{(\operatorname{ker} T)^{\perp}}$ is skew symmetric.

Proof. Since $T$ is normal, we have $\operatorname{ker} T=\operatorname{ker} T^{*}$, and $T$ can be written as

$$
T=\left[\begin{array}{ll}
0 & 0 \\
0 & A
\end{array}\right] \quad \begin{gathered}
\operatorname{ker} T \\
(\operatorname{ker} T)^{\perp}
\end{gathered} .
$$

Then the sufficiency is obvious. We need only prove the necessity.

Suppose that $T$ is skew symmetric and $C T C=-T^{*}$ for some conjugation $C$. It follows that $C(\operatorname{ker} T)=\operatorname{ker} T^{*}=\operatorname{ker} T$, and hence $C$ can be written as

$$
C=\left[\begin{array}{cc}
C_{1} & 0 \\
0 & C_{2}
\end{array}\right] \quad \begin{gathered}
\operatorname{ker} T \\
(\operatorname{ker} T)^{\perp}
\end{gathered}
$$

A simple calculation shows that $C_{2}$ is a conjugation on $(\operatorname{ker} T)^{\perp}$ and $C_{2} A C_{2}=-A^{*}$. Hence $A$ is skew symmetric.

Theorem 2.2. Let $T \in \mathcal{B}(\mathcal{H})$ be normal. Assume that $\sigma=\Sigma \cap \sigma(T)$ and $C$ is a conjugation on $\mathcal{H}$ such that $C T C=-T^{*}$. Then $C\left(\operatorname{ran} E_{T}(\sigma)\right)=\operatorname{ran} E_{T}(-\sigma)$.

Proof. Since $T$ is skew symmetric, we have $\sigma(T)=-\sigma(T)$. For a function $f$ defined on $\sigma(T)$, we denote $f^{*}(z)=\overline{f(-z)}$ for $z \in \sigma(T)$. Then $f^{*}$ is still a function on $\sigma(T)$. For $g(z)=\alpha z^{n} \bar{z}^{m}$, it is easy to see that

$$
C g(T) C=C\left(\alpha T^{n}\left(T^{*}\right)^{m}\right) C=\bar{\alpha}\left(-T^{*}\right)^{n}(-T)^{m}=g^{*}(T) .
$$

It follows that $C f(T) C=f^{*}(T)$ for each bivariate polynomial $f(z)=p(z, \bar{z})$. Since all bivariate polynomials are uniformly dense in $C\left(\sigma(T)\right.$ ) (the $C^{*}$-algebra of all complex continuous functions on $\sigma(T)$ ), one can easily deduce that $C f(T) C=$ $f^{*}(T)$ for all $f \in C(\sigma(T))$.

Obviously, there exists a sequence $\left\{f_{n}\right\}_{n=1}^{\infty}$ of continuous functions on $\sigma(T)$ such that $\sup \left\{\left|f_{n}(z)\right|: n \in \mathbb{N}, z \in \sigma(T)\right\}<\infty$ and $f_{n}(z) \rightarrow \chi_{\sigma}(z)$ for all $z \in \sigma(T)$, where $\chi_{\sigma}(z)$ is the characteristic function of $\sigma$. Then $f_{n}^{*}(z) \rightarrow \chi_{\sigma}^{*}(z)=\chi_{-\sigma}(z)$ for all $z \in \sigma(T)$. Thus $f_{n} \rightarrow \chi_{\sigma}$ and $f_{n}^{*} \rightarrow \chi_{\sigma}^{*}$ in the weak*-topology of $L^{\infty}(\mu)$, where $\mu$ is the scalar spectral measure corresponding to $T$.

By the functional calculus for normal operators ([3, Theorem 8.10]), one obtains $f_{n}(T) \rightarrow \chi_{\sigma}(T)$ and $f_{n}^{*}(T) \rightarrow \chi_{-\sigma}(T)$, both in the weak operator topology. It follows that $C \chi_{\sigma}(T) C=\chi_{-\sigma}(T)$, that is, $C E_{T}(\sigma) C=E_{T}(-\sigma)$. Thus we conclude that $C\left(\operatorname{ran} E_{T}(\sigma)\right)=\operatorname{ran} E_{T}(-\sigma)$.

Now we give the proof of Theorem 1.10 . 
Proof of Theorem 1.10, By Lemma 2.1, without loss of generality, we may directly assume that $\operatorname{ker} T=\{0\}$.

"(i) $\Longrightarrow\left(\right.$ ii)." Assume that $D$ is a conjugation on $\mathcal{H}$ such that $D T D=-T^{*}$. Denote $\sigma=\Sigma \cap \sigma(T)$. Obviously, $\sigma \cup(-\sigma)=\sigma(T) \backslash\{0\}$ and $\operatorname{ran} E_{T}(\sigma)$, ran $E_{T}(-\sigma)$ both reduce $T$. Note that $\operatorname{ker} T=\{0\}$. Then $E_{T}(\{0\})=0$ and $\operatorname{ran} E_{T}(\sigma) \oplus$ $\operatorname{ran} E_{T}(-\sigma)=\mathcal{H}$. Hence $T$ can be written as

$$
T=\left[\begin{array}{cc}
N & 0 \\
0 & B
\end{array}\right] \quad \begin{gathered}
\operatorname{ran} E_{T}(\sigma) \\
\operatorname{ran} E_{T}(-\sigma)
\end{gathered} .
$$

It is easy to see that $E_{N}(\mathbb{C} \backslash \Sigma)=0$. By Theorem 2.2, we have $D\left(\operatorname{ran} E_{T}(\sigma)\right)=$ ran $E_{T}(-\sigma)$. Hence $D$ can be written as

$$
D=\left[\begin{array}{cc}
0 & F^{-1} \\
F & 0
\end{array}\right] \quad \begin{gathered}
\operatorname{ran} E_{T}(\sigma) \\
\operatorname{ran} E_{T}(-\sigma)
\end{gathered},
$$

where $F: \operatorname{ran} E_{T}(\sigma) \rightarrow \operatorname{ran} E_{T}(-\sigma)$ is conjugate-linear, invertible and satisfies that $\langle F x, F y\rangle=\langle y, x\rangle$ for all $x, y \in \operatorname{ran} E_{T}(\sigma)$.

Since $D T D=-T^{*}$, a short calculation shows that $F N F^{-1}=-B^{*}$ and $B=$ $-F N^{*} F^{-1}$. It follows that $T=N \oplus\left(-F N^{*} F^{-1}\right)$. By Theorem 2.2, we have dim $\operatorname{ran} E_{T}(\sigma)=\operatorname{dim} \operatorname{ran} E_{T}(-\sigma)$. Then there exists a unitary operator $U$ : $\operatorname{ran} E_{T}(-\sigma) \rightarrow \operatorname{ran} E_{T}(\sigma)$ such that $U\left(\operatorname{ran} E_{T}(-\sigma)\right)=\operatorname{ran} E_{T}(\sigma)$. Define $W=$ $U F$. It is easy to see that $W$ is an antiunitary operator on $\operatorname{ran} E_{T}(\sigma)$ and

$$
T=N \oplus\left(-F N^{*} F^{-1}\right) \simeq N \oplus\left(-W N^{*} W^{-1}\right) .
$$

Denote $\mathcal{K}=\operatorname{ran} E_{T}(\sigma)$. Since $N$ is normal, there exists a conjugation $C$ on $\mathcal{K}$ such that $C N C=N^{*}$. Set $V=W C$. It is easy to verify that $V \in \mathcal{B}(\mathcal{K})$ is unitary. Thus we have

$$
T \simeq N \oplus\left(-W N^{*} W^{-1}\right)=N \oplus\left(-V C N^{*} C V^{-1}\right) \simeq N \oplus(-N) .
$$

"(ii) $\Longrightarrow$ (iii)." This is obvious.

"(iii) $\Longrightarrow($ i)." By Proposition 1.9, this is clear.

If $\mu$ is a Borel measure on $\mathbb{C}$, then we denote by $\mu^{-}$the Borel measure on $\mathbb{C}$ defined by

$$
\mu^{-}(\sigma)=\mu(-\sigma)
$$

Obviously, $\mu+\mu^{-}$is a Borel measure on $\mathbb{C}$ and $\left(\mu+\mu^{-}\right)(\sigma)=\left(\mu+\mu^{-}\right)(-\sigma)$ for any Borel subset $\sigma$ of $\mathbb{C}$.

Proposition 2.3. Let $\mu$ be a positive Borel measure with compact support, $\mu(\mathbb{C} \backslash \Sigma)=0$ and $C$ be a conjugation on $L^{2}(\mu)$. If $M_{1}$ and $M$ are respectively the "multiplication by $z$ " operator on $L^{2}(\mu)$ and $L^{2}\left(\mu+\mu^{-}\right)$, then $M_{1} \oplus\left(-M_{1}\right) \simeq M$.

Proof. Define $U: L^{2}(\mu) \oplus L^{2}(\mu) \rightarrow L^{2}\left(\mu+\mu^{-}\right)$as

$$
U(f, g)(z)= \begin{cases}f(z), & z \in \Sigma, \\ g(-z), & z \in-\Sigma .\end{cases}
$$

Since $\mu(\mathbb{C} \backslash \Sigma)=0$, one can easily verify that $U$ is unitary and $U\left(M_{1} \oplus\left(-M_{1}\right)\right) U^{*}=$ $M$.

Lemma 2.4 ([3], Theorem 10.16). If $N$ is a normal operator on $\mathcal{H}$, then there are mutually singular measures $\mu_{\infty}, \mu_{1}, \mu_{2}, \ldots$ (some of which may be zero) such that

$$
N \simeq N_{\mu_{\infty}}^{(\infty)} \oplus N_{\mu_{1}} \oplus N_{\mu_{2}}^{(2)} \oplus \cdots
$$


Proof of Theorem 1.11, "£". Let $j(1 \leq j \leq \infty)$ be fixed. Define a map $C_{j}$ on $L^{2}\left(\mu_{j}\right)$ by $\left(C_{j} f\right)(z)=\overline{f(-z)}$. Since $\mu_{j}(\sigma)=\mu_{j}(-\sigma)$ for each Borel subset $\sigma$ of $\mathbb{C}$, one can deduce that $C_{j}$ is well defined and $C_{j}$ is a conjugation on $L^{2}\left(\mu_{j}\right)$. A direct calculation shows that

$$
\left(N_{j} C_{j} f\right)(z)=z \overline{f(-z)}=-\left(C_{j} N_{j}^{*} f\right)(z), \forall f \in L^{2}\left(\mu_{j}\right) .
$$

This means that $C_{j} N_{j} C_{j}=-N_{j}^{*}$ and $N_{j}$ is skew symmetric. Thus

$$
T \simeq \bigoplus_{1 \leq j \leq \infty} N_{j}^{(j)}
$$

is skew symmetric.

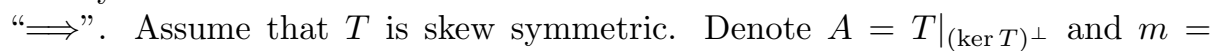
$\operatorname{dim} \operatorname{ker} T$. By Lemma2.1, $A$ is a skew symmetric normal operator with $\operatorname{ker} A=\{0\}$ and $T \simeq M_{z}^{(m)} \oplus A$, where $M_{z}$ is the "multiplication by $z$ " operator on $L^{2}(\nu)$ with $\nu$ defined by $\nu(\{0\})=1$ and $\nu(\mathbb{C} \backslash\{0\})=0$.

Since $A$ is skew symmetric, by Theorem 1.10 one can deduce that

$$
A \simeq N \oplus(-N),
$$

where $N$ is a normal operator on some Hilbert space $\mathcal{K}$ with $E_{N}(\mathbb{C} \backslash \Sigma)=0$.

By Lemma 2.4 $N$ can be written as

$$
N=\left[\begin{array}{cccc}
N_{\nu_{\infty}}^{(\infty)} & & & \\
& N_{\nu_{1}} & & \\
& & N_{\nu_{2}}^{(2)} & \\
& & & \ddots
\end{array}\right] \begin{gathered}
\mathcal{K}_{\infty}^{(\infty)} \\
\mathcal{K}_{1} \\
\mathcal{K}_{2}^{(2)} \\
\vdots
\end{gathered}
$$

where $\mathcal{K}_{j}=L^{2}\left(\nu_{j}\right)(1 \leq j \leq \infty), \nu_{\infty}, \nu_{1}, \nu_{2}, \cdots$ (some of which may be zero) are mutually singular, finitely supported Borel measures on $\mathbb{C}$. In view of $E_{N}(\mathbb{C} \backslash \Sigma)=$ 0 , we obtain $\nu_{j}(\mathbb{C} \backslash \Sigma)=0$ for all $1 \leq j \leq \infty$.

By Proposition [2.3, it follows that $N_{\nu_{j}} \oplus\left(-N_{\nu_{j}}\right) \simeq M_{j}$ for each $j$, where $M_{j}$ is the "multiplication by $z$ " operator on $L^{2}\left(\nu_{j}+\nu_{j}{ }^{-}\right)$. For $1 \leq j \leq \infty$, denote $\theta_{j}=\nu_{j}+\nu_{j}{ }^{-}$. Then $\theta_{j}(\sigma)=\theta_{j}(-\sigma)$ for any $j$ and any Borel subset $\sigma$ of $\mathbb{C}$. Thus we have proved that

$$
A \simeq \bigoplus_{1 \leq j \leq \infty} M_{j}^{(j)}
$$

where $M_{j}$ is the "multiplication by $z$ " operator on $L^{2}\left(\theta_{j}\right)(1 \leq j \leq \infty)$.

For $1 \leq j \leq \infty$, set

$$
\mu_{j}= \begin{cases}\theta_{j}, & j \neq m \\ \theta_{j}+\nu, & j=m\end{cases}
$$

Then $\mu_{m}=\theta_{m}+\nu$ is a Borel measure on $\mathbb{C}$. It is easy to see that $\mu_{j}(\sigma)=\mu_{j}(-\sigma)$ for any $1 \leq j \leq \infty$ and any Borel subset $\sigma$ of $\mathbb{C}$. Note that $\theta_{m}$ and $\nu$ are mutually singular. Thus we have

$$
T \simeq M_{z}^{(m)} \oplus A \simeq\left(M_{z}^{(m)} \oplus M_{m}^{(m)}\right) \oplus\left(\bigoplus_{j \neq m} M_{j}^{(j)}\right) \simeq \bigoplus_{1 \leq j \leq \infty} N_{j}^{(j)},
$$

where $N_{j}$ is the "multiplication by $z$ " operator on $L^{2}\left(\mu_{j}\right)(1 \leq j \leq \infty)$. This completes the proof. 


\section{REFERENCES}

1. P. Benner, R. Byers, V. Mehrmann, and H. G. Xu, Numerical computation of deflating subspaces of skew-Hamiltonian/Hamiltonian pencils, SIAM J. Matrix Anal. Appl. 24 (2002), no. 1, 165-190. MR.1920561 (2003f:49068)

2. J. A. Cima, S. R. Garcia, W. T. Ross, and W. R. Wogen, Truncated Toeplitz operators: Spatial isomorphism, unitary equivalence, and similarity, Indiana Univ. Math. J. 59 (2010), no. 2, 595-620. MR2648079 (2011i:47035)

3. J. B. Conway, A course in functional analysis, second ed., Graduate Texts in Mathematics, vol. 96, Springer-Verlag, New York, 1990. MR.1070713 (91e:46001)

4. F. R. Gantmacher, The theory of matrices. Vol. 2, translated by K. A. Hirsch, Chelsea Publishing Co., New York, 1959. MR0107649 (21:6372c)

5. S. R. Garcia, Aluthge transforms of complex symmetric operators, Integral Equations Operator Theory 60 (2008), no. 3, 357-367. MR2392831 (2008m:47052)

6. S. R. Garcia and M. Putinar, Complex symmetric operators and applications, Trans. Amer. Math. Soc. 358 (2006), no. 3, 1285-1315 (electronic). MR2187654 (2006j:47036)

7. S. R. Garcia and M. Putinar, Complex symmetric operators and applications. II, Trans. Amer. Math. Soc. 359 (2007), no. 8, 3913-3931 (electronic). MR2302518 (2008b:47005)

8. S. R. Garcia and M. Putinar, Interpolation and complex symmetry, Tohoku Math. J. (2) 60 (2008), no. 3, 423-440. MR2453732 (2009k:47048)

9. S. R. Garcia and W. R. Wogen, Complex symmetric partial isometries, J. Funct. Anal. 257 (2009), no. 4, 1251-1260. MR2535469 (2011g:47005)

10. S. R. Garcia and W. R. Wogen, Some new classes of complex symmetric operators, Trans. Amer. Math. Soc. 362 (2010), no. 11, 6065-6077. MR2661508 (2011g:47086)

11. L.-K. Hua, On the theory of automorphic functions of a matrix variable. II. The classification of hypercircles under the symplectic group, Amer. J. Math. 66 (1944), 531-563. MR.0011134 $(6: 124 \mathrm{c})$

12. L.-K. Hua, On the theory of automorphic functions of a matrix level. I. Geometrical basis, Amer. J. Math. 66 (1944), 470-488. MR0011133 (6:124b)

13. V. Mehrmann and D. Watkins, Polynomial eigenvalue problems with Hamiltonian structure, Electr. Trans. Num. Anal. 13 (2002), 106-113. MR1961201(2004e:65046)

14. V. Mehrmann and H. Xu, Numerical methods in control, J. Comput. Appl. Math. 123 (2000), 371-394. MR1798534 (2002i:93045)

15. N. H. Scott, A new canonical form for complex symmetric matrices, Proc. Roy. Soc. London Ser. A 441 (1993), no. 1913, 625-640. MR1225775 (94e:15029)

16. D. Sorensen, Passivity preserving model reduction via interpolation of spectral zeros, Systems and Control Letters 54 (2005), 347-360. MR2123708 (2005i:93021)

17. S. M. Zagorodnyuk, On a J-polar decomposition of a bounded operator and matrices of $J$-symmetric and J-skew-symmetric operators, Banach J. Math. Anal. 4 (2010), no. 2, 11-36. MR2606479 (2011f:47032)

18. S. M. Zagorodnyuk, On the complex symmetric and skew-symmetric operators with a simple spectrum, Symmetry, Integrability and Geometry: Methods and Applications 7 (2011), 1-9. MR2804580(2012f:47006)

19. S. Zhu and C. G. Li, Complex symmetric weighted shifts, Trans. Amer. Math. Soc. 365 (2013), no. 1, 511-530. MR2984066

20. S. Zhu, C. G. Li, and Y. Q. Ji, The class of complex symmetric operators is not closed, Proc. Amer. Math. Soc. 140 (2012), no. 5, 1705-1708. MR2869154

Institute of Mathematics, Jilin University, Changchun 130012, People's Republic of CHINA

E-mail address: licg09@mails.jlu.edu.cn

Department of Mathematics, Jilin University, Changchun 130012, People's Republic OF CHINA

Current address: School of Mathematical Sciences, Fudan University, 220 Handan Road,

Shanghai 200433, People's Republic of China

E-mail address: zhusen@jlu.edu.cn 